\title{
Fachliche Praxis und die fachliche Prägung gesellschaftlicher Interaktion
}

\author{
Ludwig M. EICHINGER
}

\begin{abstract}
Professional and technical practice and the technical character of social interaction

The focus on communication in research on professional and scientific language somehow reflects the intention of John L. Austin's phrase "How to do things with words?" But a description based on the concept of communication ultimately also relies on linguistic idiosyncrasies. We will look at things the other way round and ask first "how to do (professional) things" and then look at the linguistic units used specifically for this purpose. Professionalism in this view takes very different forms for different types of actions ("practices"). Although reliability and professional authority are central features of all linguistic realizations to be considered, they are represented in very different ways. As a result, professionalism not only shows in the high degree of explicitness of technical prose typical for written scientific discussion. It is also reflected in the high degree of implicitness of speech that accompanies and constitutes practical action.
\end{abstract}

Keywords: specialist communication, social practice, orality, professional knowledge

DOI: doi.org/10.15452/StudiaGermanistica.2020.26.0002

\section{Fachsprache, Fachkommunikation, fachliche Interaktion}

Schon vor langem ist man in der Diskussion um die Sprache in fachlichen Kontexten den Schritt von der Untersuchung der Fachsprache zu der der Eigenheiten von Fachkommunikation gegangen. Bereits in den um die Wende zum 21. Jahrhundert erschienenen repräsentativen HSK-Bänden ,Fachsprachen' finden sich Feststellungen, in denen der Status der titelgebenden Fachsprache relativiert wird:

(1) „Dieser Stand der aktuellen Fachsprachenforschung umgreift inzwischen eine Komplexität von den textinternen sprachlichen bis zu den textexternen pragmatischen Komponenten, was inzwischen das Forschungsinteresse von den Systemeigenschaften der Fachsprachen als Subsprachen, die in ihrer Mehrheit erkannt sind, zunehmend verlagert hat zu den Verwendungseigenschaften. Dies läßt immer mehr angeraten erscheinen, statt von ,Fachsprachen' als dem Medium und von ,Fachtexten' als den Manifestationen doch eher von ,Fachkommunikation' zu sprechen, wenn das Mittel fachlicher Interaktion wie auch der Forschungsgegenstand selbst gemeint sind. Man kann auch die Formel ,Fachsprachen in der Fachkommunikation“ wählen.“ (Hoffmann/Kalverkämper 1998:358) 
Der Schritt, der damit vollzogen ist, ist einer auf dem Weg von der Betrachtung des innerfachlichen Codes hin zu einer Charakteristik der sprachlichen Haltung in einer Wissensgesellschaft. Wenn man die weitere Wegstrecke zu diesem recht abstrakt klingenden Ziel hin konkretisieren will, sollte man den Schritt von der Fachkommunikation hin zu den fachlichen Weisen des Handelns in der heutigen Welt machen, hin zur fachlichen Interaktion (s. dazu Deppermann 2018:54). Unter fachlicher Interaktion soll das Agieren im Rahmen als fachlich betrachteter Praktiken gelten, denen Sprachspiele zugeordnet werden, die in diesem Kontext als fachlich adäquates sprachliches Handeln verstanden werden. Was könnte das heißen?

Es handelt sich bei dieser Art, die Dinge zu sehen, um eine Umkehrung des Blicks im Verhältnis zur traditionellen Sichtweise, die das Fachliche in den sprachlichen Formen und Kommunikationstypen sucht. Wenn sprachliche Äußerungen als alltagssprachlich, gruppensprachlich usw. und eben auch fachsprachlich bezeichnet werden, geht es aber um den Ort solcher Äußerungen im Spektrum gesellschaftlicher Praktiken insgesamt. Damit soll die Bedeutung der Sprache als des explizitesten Mittels der Interaktion nicht geringgeschätzt werden. Praktiken ihrerseits sind Handlungszusammenhänge, die auch durch sprachliche Muster und Sprachspiele konstituiert werden (s. Eichinger 2016:IX). Fachlichkeit ist dann zunächst eine Eigenschaft von bestimmten Praktiken, für die im Prinzip die gleichen Bedingungen der Verlässlichkeit gelten, wie sie zum Gelingen von Kommunikation insgesamt beitragen, nämlich so etwas wie eine Kombination von Können, Wissen, wahren Überzeugungen und gegebenenfalls explizierbaren Gründen (s. dazu Brandom 2001:156-157; Wittgenstein 1971:87 [Nr. 337] und 126 [Nr. 484]). Was als fachliche Praxis gilt, ist eine Frage von gesellschaftlichen Übereinkünften und Festlegungen. Die sind nicht trennscharf in ihrer Abgrenzung gegenüber andersartigen Praktiken und sie können sich natürlich verändern. So sind auch die sprachlichen Erscheinungen, die sich dort finden, nicht trennscharf von denen unterschieden, die in anderen Domänen („Praxisräumen“) auftauchen, und auch die Bewertung als „fachsprachlich“ mag variieren. Diese Verbindung von sprachlich insgesamt Gängigem in spezifischer Konstellation und mit einem Kern von Eigenheiten kennzeichnet ja ganz generell gesellschaftlich bedingte Variation im Sprachgebrauch.

\section{Das eine Ende: Schriftliche Interaktion in der Wissenschaft}

\subsection{Komplexität}

In wissenschaftlichen Texten als den zentralen Elementen einer zwischen Fachleuten stattfindenden schriftlichen wissenschaftssprachlichen Interaktion findet sich häufig eine so deutliche Häufung von Indizien für Fachlichkeit, dass sie oft auf den ersten Blick unstrittig dem Kernbereich fachlicher Praktiken zugeordnet werden. Sie lassen sich lesen als Signale einer entpersonalisierten und situationsentbundenen Kommunikation mit den daraus folgenden Notwendigkeiten, die Bedingungen der Kommunikation in jedem Schritt klarer zu machen, als wir das im sprachlichen Alltag tun und tun würden. Dazu gehört, dass die Vorgaben für diese Texttypen strikter sind, dass sie außerdem im innersten Kern zunächst auf die bei Karl Bühler (1999 [1934]) titelgebende Darstellungsfunktion der Sprache bezogen sind. Diese schriftsprachliche Praxis der Interaktion nutzt daher in hohem Maße Strukturen von Skripturalität und ist durch einen hohen Grad an Explizitheit gekennzeichnet, beides Merkmale von Distanzkommunikation, mit denen die Dominanz der Darstellungsfunktion signalisiert wird (zum theoretischen Hintergrund s. Ágel 2015:124 und passim). Gegenüber den umfassenden Möglichkeiten einer Sprache steckt darin eine - bewusste - versachlichende Reduktion (s. resümierend Taylor 2016: 88/89). Dass sie damit eigentlich eher einen Sonderfall als den Zentralfall sprachlicher Praktiken darstellen, wird von Angelika Linke (2015:33) hervorgehoben: die „Fähigkeit zu ,monologistischer" Rede“ habe ,als (evolutionär) späte Ausprägung menschlicher Sprachlichkeit“ zu gelten. Andererseits bleibt festzuhalten, dass die Entwicklung dieses Texttyps für die europäische Gesellschaftsgeschichte unter den Auspizien einer Welt der gedruckten Texte und wachsender Öffentlichkeiten von zentraler Bedeutung ist (vgl. Giesecke 1998:512/513; s. insgesamt auch Ágel 2015).

Um an dieser (speziellen) Interaktion in angemessener Weise teilnehmen zu können, sind zudem die höchsten Ansprüche an Kenntnis im Fachlichen wie an dessen sprachlicher Fassung zu 
erfüllen. Schon der Ort der Texte - typisch: der Text erscheint in einer referierten Fachzeitschrift eines Faches - macht klar, dass es sich um einen Beitrag im Rahmen eines Faches handelt. Normalerweise muss man, um auf dieser Ebene teilnehmen zu können, in solch ein Fach professionell eingeführt und auch darin bereits weiter qualifiziert sein. Das betrifft die inhaltliche Kompetenz, aber auch die Meisterung der passenden sprachlichen Form in den Traditionen des Faches, damit betrifft es auch ihren bewussten Bruch, den man sich wegen seiner deutlichen Signalwirkung aber sozialsymbolisch leisten können muss. Am Übergang zwischen der fachlichen und der sprachlich-stilistischen Kompetenz steht die Beherrschung der einschlägigen Terminologie bzw. nicht so strikt terminologisch definierten Fächer, der Konventionen der Begriffsbildung. Die dabei genutzten Techniken lassen sich durchwegs als Strategien verstehen, den Erwartungen an die Explizitheit und an die erfolgende Reduktion von Komplexität zu entsprechen. Solche Texte sehen dann - in einem geisteswissenschaftlichen Kontext - zum Beispiel wie der folgende aus, ein Text, der typische formale Merkmale solch eines Interaktionstyps im Deutschen sowohl benennt wie in bestimmter Hinsicht selbst demonstriert (vgl. Eichinger 2010:157-162):

(2) „Über die Bindung an die jeweilige alltägliche Sprache ist die Wissenschaftssprache auch an deren strukturelle Vorgaben gebunden. Sie ist darauf angewiesen - kann sie zugleich im Rahmen des sprachentwicklungsmäßig Möglichen freilich auch weiter entfalten helfen.

Um dies am Beispiel des Deutschen zu illustrieren, sind hier für die Wissenschaftssprache Strukturmerkmale wie die Entwicklung einer spezifischen syntaktischen Komplexität oder etwa die Nominalbildungsmechanismen zu nennen. Das Englische wiederum bietet in den Serialisierungsmöglichkeiten und -erfordernissen Vorgaben, die sich in der Wissenschaftskommunikation um- und durchsetzen.“ (Ehlich 2006:21)

Es sei hier nur auf die Elemente nominaler Integration hingewiesen, die den Text entscheidend prägen, etwa Nominalgruppen, wie die um den Kern Strukturmerkmale im zweiten Absatz, oder das tatsächlich selbstreferentielle dreigliedrige Kompositum Nominalbildungsmechanismen. In ihm fällt auch schon jenes Element auf, in solchen Komposita nicht so sehr Untertypen - hier von Mechanismen - zu benennen, vielmehr den eigentlich inhaltlichen Kern - hier Nominalbildung - in eine abstraktere Kategorie einzuordnen. Der gleiche Effekt zeigt sich bei der Entfaltung von Serialisierung im Hinblick auf Möglichkeiten und Erfordernisse im letzten Satz. Gerade diese formal recht implizite Einordnung in die Kategorien und Wissensordnungen reduziert innerfachlich die Komplexität, insofern auf übergeordnete gängige Strukturen verwiesen wird.

Was hier innerfachlich ökonomisch ist, erhöht aber die Komplexität für die nicht entsprechend sozialisierten Leser. Was hier am Beispiel eines hoch integrativen Stilmerkmals angedeutet wurde, gilt natürlich noch verstärkt bei der Nutzung von Termini. Wie weit die ,Verkapselung“ der Komplexität in Termini geht, ist je nach Wissenschaftsbereich unterschiedlich (in hoch terminologisierten Fächern der Lebens-, Natur- und Technikwissenschaften logischerweise deutlich mehr als bei den Sozialwissenschaften und den Geisteswissenschaften). Hier spielen auch die noch „neutraleren“ Darstellungsformen wie Formeln, Graphen, Tabellen und insgesamt Visualisierungen eine strukturbildende Rolle. Das hat logischerweise enorme Konsequenzen für die auf dieser Ebene erwartete Komplexität der syntaktischen Oberfläche, wie man an dem folgenden Text (3) deutlich sieht. Naturwissenschaftliche Texte dieser Art zeigen prinzipiell eine eher einfache syntaktische Struktur mit viel Terminologie. Bemerkenswert ist auch hier der hohe Grad an nominaler Integration. Verbal sind lediglich das unpersönliche Verb es kommt zu und die Nominalverbfügung die Klassifikation erfolgt. Die inhaltlichen Frames werden von den Nominalisierungen Hydroxylierung und Konzentrationsanstieg bzw. eben Klassifikation gesetzt. Man kann diese Punkte und weitere dem folgenden kurzen Ausschnitt unmittelbar entnehmen, es soll nicht im Einzelnen analysiert werden.

(3) Aufgrund der gestörten Hydroxylierung von Phenylalanin (L-Phe) zu Tyrosin (L-Tyr) kommt es zu einem Konzentrationsanstieg der essentiellen Aminosäure Phenylalanin und einiger Metaboliten (wie zum Beispiel Phenylpyruvat, Phenylacetat und Phenyllactat) im Blut und in weiteren Körperkompartimenten (Kaufman 1989). 


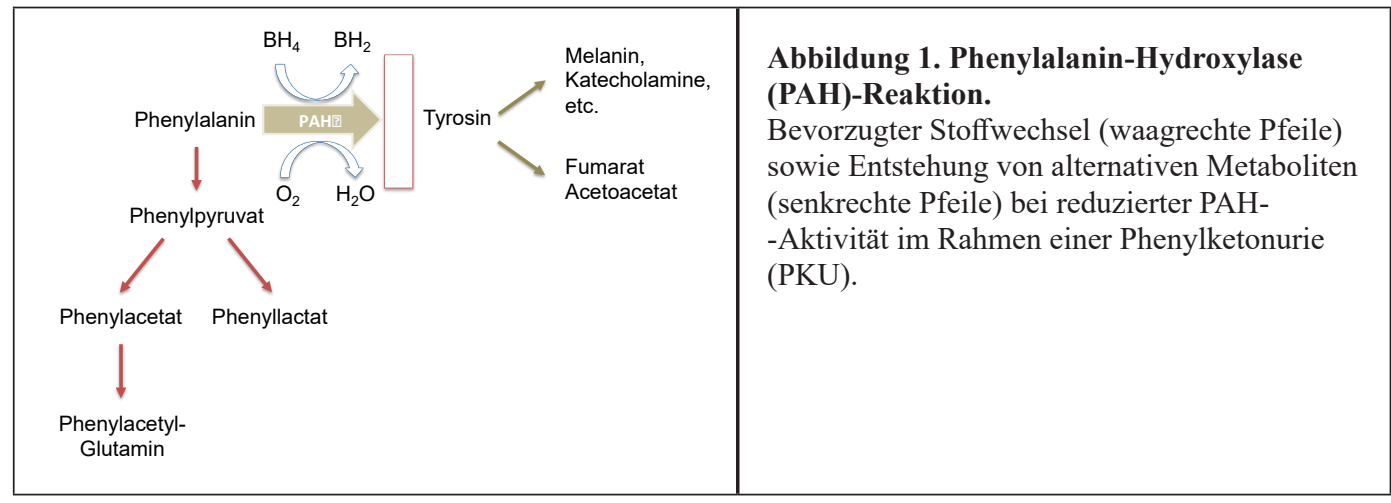

Die Klassifikation der Erkrankung erfolgt historisch anhand der Phenylalaninkonzentration im Plasma zum Zeitpunkt der Diagnosestellung und damit vor Beginn therapeutischer Maßnahmen: Klassische PKU mit [Phe] > $1200 \mu \mathrm{mol} / \mathrm{l}$ (> $20 \mathrm{mg} / \mathrm{dl})$, Milde PKU mit [Phe] $600-1200 \mu \mathrm{mol} / \mathrm{l}(10-20$ $\mathrm{mg} / \mathrm{dl}$ ) und Hyperphenylalaninämie (HPA) mit Phenylalaninkonzentrationen von 120 - $600 \mu \mathrm{mol} / \mathrm{l}$ $(2-10 \mathrm{mg} / \mathrm{dl}$ ) (Williams et al. 2008). Eichinger, Anna (2018): Charakterisierung eines Mausmodells zur BH4-sensitiven Phenylketonurie. Dissertation. München: LMU, S. 1)

\subsection{Dialog}

Trotz ihrer formalen Abgeschlossenheit als Texte sind Fachtexte aber doch auch als Teil der interaktionellen Praxis eines Dialogs mit entsprechend kompetentem Partner konzeptualisiert. Das erlaubt Verdichtungen und Verkürzungen, die mit der Dekodierungskompetenz der möglichen Rezipienten rechnet - und die sich an den Tendenzen zur Implizitheit und damit zur formalen Verdichtung zeigen, die wir an den besprochenen Beispielen gesehen haben. Was aber das grundsätzlich Dialogische dieser Texte angeht, so rechnet jeder Text damit, eine Antwort zu finden, nicht nur in dazu dezidiert geschaffenen Text-Modellen des Aufeinander-Antwortens, wie es zum Beispiel Rezensionen oder in gewissem Umfang Fußnoten sind. Diese angenommene Dialogizität schlägt sich in der Komplexität der Darstellung nieder. Häufig reicht auf der Ebene der Begründungen nicht hin, einfach die Gründe für die gemachten Aussagen zu geben, vielmehr müssen bekannte Gegengründe abgewehrt bzw. mögliche Gegengründe präsumtiv beantwortet werden. Einen Reflex dieser Bedingungen stellen die Literaturverweise bzw. die Einordnungen in Fußnoten dar. Bekanntlich ist es an dieser Stelle von Fachtraditionen abhängig, welche historische Tiefe dieser virtuelle Dialog annimmt. Und es hat Folgen für die präferierten Publikationsformen: so ist in den Naturwissenschaften das möglichst häufig erscheinende wissenschaftliche Journal und der ,preprint" die Form, die dieser Art von Interaktion angemessen erscheint (zudem gerne in dem internationalen Englisch und mit viel Visualisierung). Im Umfeld IT-orientierter Fächer schlägt sich das darin nieder, dass der akzeptierte Tagungsbeitrag, auch das dazugehörige abstract als die am höchsten eingeschätzten Publikationen gelten. Am anderen Ende stehen logischerweise die Geisteswissenschaften und jedenfalls Teile der Sozialwissenschaften, wo die Darstellung der eigenen fachlichen Behauptungen viel natürlichsprachigen Raum einnimmt, so dass die potentielle Dialogizität deutlich zurücktritt, am klarsten bei der textuellen „Königsdisziplin“, der wissenschaftlichen - nicht lehrbuchartigen - Monographie, Paradebeispiel für eine Interaktion in längeren zeitlichen Wellen (vgl. AvH 2009). Hier beruhen die Merkmale der sprachlichen Struktur auf den Traditionen der Wissenskonstitution und -vermittlung in einer entwickelten und „monomodalen“ Schriftlichkeit und Skripturalität einer Welt, die nichts als Wörter und Buchstaben zu kennen scheint, und hier eine ganze Folge von Bezügen aufweist. 


\subsection{Explizitheit und Komplexität}

Resümierend kann man sagen, jener unbestrittene Kern von Fachlichkeit, wie ihn z. B. die geschriebene Wissenschaftssprache darstellt, ist die Ebene der fachlichen Interaktion, in der es darum geht, die Komplexität des Gegenstands möglichst explizit aufscheinen zu lassen. Das heißt, wiewohl man den Zweck von Wissenschaft darin sehen kann, „Inseln der Stabilität im unendlichen Meer der Komplexität" der Welt zu erzeugen, wie das mein Lehrer Harald Weinrich (1973:145) in den 1970er Jahren einmal formuliert hat, erzeugt das eine lokale Erhöhung der Komplexität durch die Konstruktion eines wissenschaftlichen Subsystems (der Fachmann sieht dort mehr als der Laie). Dazu braucht es eine geregelte Begrifflichkeit, eine terminologische Festlegung bzw. zumindest eine weitgehende Diskussion um solche Fragen, die Explizitheitsanforderungen sind hoch, die Begründungsbedingungen strikt und auf möglichste Explizitheit angelegt (vgl. dazu Fandrych 2018:148-149; auch Ágel 2015:127, bes. Fn. 9). Jeder bessere Beitrag in einer wissenschaftlichen Zeitschrift oder einer wissenschaftlichen Monographie ist ein Beleg dafür. Hier ein etwas dramatisches literaturwissenschaftliches Beispiel (s. Eichinger 2005:374-375):

(4) Ich wähle im folgenden unter dem allgemeinen Gesichtspunkt der Interpretation der Natur im Rahmen menschlichen Selbstverständnisses zwei Beispiele aus der Aufklärungszeit aus und versuche, ihre gemeinhin nicht vermutete Anknüpfung oder nicht vermutete Art der Anknüpfung an Lehrstücke der Antike und des Mittelalters aufzuweisen: nämlich Rousseaus Konzept des Naturgesetzes im 2. „Discours“ und Kants Argument gegen einen naturalistischen Selbsthaß der Vernunft zu Beginn seiner Grundlegung der Metaphysik der Sitten, beides bezogen auf die stoische Oikeiosislehre und ihre Verarbeitung durch Thomas von Aquin. (Forschner, Maximilian (1990): Über natürliche Neigungen. In: Bubner, Rüdiger u.a. (Hrsg.): Die Trennung von Natur und Geist. München, S. 95).

Der Explizitheitsanspruch findet seine Grenzen in dem Wissen darüber, was ein anzunehmender gemeinsamer Nenner ist, wenn man überhaupt an diesem interaktionalen Spiel teilnehmen will. So steckt hinter der komplexen Verschachtelung, vor allem in den verschiedenen Attributen, eine Wissenswelt, auf die nur implizit verwiesen wird. Die ganze zweite Hälfte dieses Textausschnitts stellt eine appositive Fügung und Entfaltung der zu Beginn angekündigten beiden Beispiele dar, in Form von zwei Substantivgruppen, wo vor allem in der zweiten eine Vielfalt von Beziehungen in integrierter Form erscheint, so dass der Leser einerseits die propositionale Entfaltung selbst leisten muss, wenn er denn aufgrund seines Vorwissens in der Lage ist, daraus Sinn zu machen. Nicht einfacher wird es dadurch, dass dann noch eine auf beide Substantivgruppen bezogene partizipiale appositive Konstruktion angefügt wird - eine im Deutschen recht markierte Konstruktion. Die textuelle Erscheinung in einer nicht direkt syntaktisch verdichteten integrierten Form verweist darauf, dass im Prinzip erwartet wird, der Leser könne die propositionalen Lücken füllen, bzw. zumindest den gemeinten Rahmen des Diskurses rekonstruieren, so dass solche integrierten Formen in diesem Rahmen als verdichtete Ankündigung von Neuem verstanden werden können. Auch dadurch, dass hier eine Vertrautheit mit den Traditionen fachlichen Sprechens und ein professioneller Umgang mit ihnen vorausgesetzt wird, präsentiert sich das intendierte Sprachspiel als eine fachliche Praktik, damit auch sprachliche eines exklusiven Subsystems, in dem die professionell betriebenen Fächer und Wissenschaften exklusive Systeme eigenen Rechts darstellen (zu entsprechenden Eigenheiten und Entwicklungen im Historischen s. Eichinger 1995; Ágel 2015:142-143). Für den kompetenten Spieler reduziert die Verdichtung den Wahrnehmungsaufwand.

Für die nicht oder nicht hinreichend mit diesem System Vertrauten erhöht diese Art von Interaktion aber den Grad an Komplexität (s. dazu Eichinger 2020; i. Dr.). In der gesellschaftlichen Interaktion wird das kritisch, zu einem Problem der Verlässlichkeit, vor allem bei Tatbeständen, die in Praktiken eingebunden sind, die gleichzeitig eine alltägliche wie fachliche Dimension haben, was ja für einen literaturwissenschaftlichen Diskurs eher nicht gilt. Ein Großteil der öffentlichen Kritik an juristischer Sprache und an Verwaltungssprache lässt sich auf diese Doppelheit der Bezugssysteme zurückführen, sie wird daher oft etwas leichthin geübt, da die Differenz der fachlichen und der alltäglichen Praktik an dieser Stelle oft nicht auf den ersten Blick sichtbar wird. Gerade ein 
funktionierendes juristisches und bürokratisches System ist einerseits auf höchstmögliche Genauigkeit, andererseits auf tiefe implizite Verweisketten angelegt. Das macht die Texte für den Laien gleichzeitig überexplizit und überimplizit, wiewohl sie den internen Umgang zwischen denen, die sich hier auskennen, eindeutig strukturieren. Das betrifft vor allem Bereiche, bei denen die jeweilige Praxis alltäglich so zugänglich ist, eben nicht als fachlich distant, so dass ihre fachliche Behandlung dem entsprechenden Alltagsdiskurs fremd und vielleicht unnötig abstrakt erscheint - etwa bei Regelungen aus dem Bauwesen, um die es im folgenden Beispiel geht. Sie indiziert schon im Titel mit seinem vierstufigen komplexen Substantiv - unterschiedlich leichter Auflösbarkeit - und der Kurzform für den „ökonomischen“ Gebrauch die Zugehörigkeit zu einer Kaskade verrechtlichter Schritte (vgl. Baumann 2018:44-45).

(5) Ablösungsbeträgeberechnungsverordnung $A B B V$

\section{$\S 1$ Anwendungsbereich}

(1) Diese Verordnung gilt für die Berechnung der zu leistenden Ablösungsbeträge nach den Vorschriften des Eisenbahnkreuzungsgesetzes, des Bundesfernstraßengesetzes und des Bundeswasserstraßengesetzes.

(2) Erhaltungskosten (Unterhaltungs- und Erneuerungskosten) im Sinne dieser Verordnung entsprechen den Erhaltungs- und Betriebskosten im Sinne des Eisenbahnkreuzungsgesetzes sowie den Unterhaltungskosten im Sinne des Bundesfernstraßengesetzes und des Bundeswasserstraßengesetzes.

\section{§ 2 Berechnung}

(1) Der Ablösungsbetrag ist durch Gegenüberstellung der kapitalisierten Erhaltungskosten der alten und neuen baulichen Anlagen gemäß der Anlage zu dieser Verordnung zu ermitteln.

(2) Sind die kapitalisierten Erhaltungskosten der neuen baulichen Anlagen höher als die für die alten baulichen Anlagen ermittelten Kosten, handelt es sich bei dem Differenzbetrag um die dem erhaltungspflichtigen Baulastträger von dem anderen Beteiligten abzulösenden Erhaltungsmehrkosten. Im umgekehrten Falle handelt es sich bei dem Differenzbetrag um den vom erhaltungspflichtigen Baulastträger dem anderen Beteiligten zu erstattenden Vorteilsausgleich.

Dann dient schon der ganze erste Paragraph der Einordnung in ein terminologisch fixiertes Benennungs- und Regelungssystem. Der zweite Paragraph regelt dann in einer rechtlich verlässlichen Weise - und das heißt unter anderem durch variationsfreie textuelle Ersetzungsketten - wie finanzielle Vor- und Nachteile in diesem speziellen Kontext abzugleichen sind, daher die vielen modalen Passiv-Ersatzformen. Es handelt sich hier von den Sachverhalten wie vom juristischen Regelungsbedarf um sehr spezielle Praktiken eines sehr spezifischen Expertenpublikums, für die daher Verlässlichkeit und Kompatibilität mit anderen entsprechenden Regelungen die zentralen Leitlinien darstellen.

Der Nutzen dieses Tatbestands zeigt sich in der möglichen Reduktion solcher Ergebnisse zu erfolgreichen Praxen, in anderen, angewandten, Subsystemen, d.h. wenn damit zum Beispiel das Bauen mit Beteiligung verschiedener Akteure eindeutiger geregelt ist. Explizites Rechts- und Verwaltungshandeln ist ein Paradebeispiel für eine Fachlichkeit, die auf komplexen sprachlichen Verweisstrukturen beruht, also zum Beispiel nicht nur in den eigenen Aussagen terminologisch spricht, sondern auch mehr oder minder explizite Hinweise auf die eigene Diskursgeschichte mit sich zieht. Die Behandlung einschlägiger Probleme auf dieser Ebene setzt bei den Interagenten voraus, dass diese sprachlichen Hinweise aufgelöst werden können. Moderne Gesellschaften in ihrer Komplexität brauchen solch eine Regelung, und wenn auf solch einer Ebene glückendes professionelles Handeln in der Welt des Alltags möglich ist - in unserem Beispiel beim Bauen -, bedarf es zwar des Wissenserwerbs aus der Regelungsebene, Fachlichkeit zeigt sich hier aber ganz anders, sie wird in Praktiken umgesetzt, in denen sich die Verlässlichkeit eher im Können zeigt, bei denen sprachliche Explizitheit über das Wissen eher hinderlich wäre, bzw. erst wichtig wird bei Komplikationen, oder auch in fachlichen Einführungssituationen. Hier wird praktisch wirksam, was oben schon einmal angedeutet wurde, dass es ein Wissen und eine Fachlichkeit gibt, die auf expliziter Begründung fußt, und ein Wissen und eine Fachlichkeit, die auf der Gewissheit professioneller fachlicher Erfahrung fußt. 


\section{Das andere Ende: Fachliches Handeln im mündlichen Umfeld}

So wie in diesem zweiten Fall sind die Verhältnisse beim gemeinsamen Arbeiten an einem praktischen Ziel. Jenes Wissen und Können, das unmittelbar ein fachlich kompetentes Handeln erlaubt, ohne in den jeweiligen Praktiken auf die expliziten Begründung angewiesen zu sein, um als verlässlich zu gelten, spielt in solchen Kontexten eine große Rolle; Brandom (2000:325) spricht hier von „nicht inferentieller Autorität“ und auch das Konzept der craftmanship bei Richard Sennett (2008:268-285 und besonders auch 179-180), das sich am Konzept der Meisterlehre niederschlägt, lässt sich in dieser Weise interpretieren. Die Frage ist, wo in dieser sprachlich wenig expliziten Welt des Handelns das Fachliche bleibt, und wie das den Sprachspielen abzulesen und den verwendeten Äußerungen zuzuordnen ist.

Was diese Art von Zusammenarbeit sprachlich absichert, wird in einer fachlichen Praxis ähnlich realisiert werden wie in einem praktischen Alltagshandeln, das wir nicht für fachlich halten würden - man wird zum Beispiel eher kein übermäßiges Maß an Explizitheit erwarten. Wie erkennt man unter diesen Umständen die Fachlichkeit einer Praktik, und welche Rolle spielt hier die Sprache? Denn zunächst einmal geht es hier um Gespräche, und so kommen Elemente ins Spiel, wie sie für Gespräche konstitutiv sind. Andererseits sollten aber die fachlichen unter diesen Praktiken, da sie sich auf komplexe Wissensbestände beziehen, nicht ohne spezifische sprachliche Möglichkeiten auskommen, die aber angemessen in die Abläufe eingebettet sein müssen. Auch wenn er nicht (oder allenfalls nur ansatzweise) expliziert wird, stehen fachliche Äußerungen in einem Diskurszusammenhang, dessen Ziel die Darstellung, Diskussion, Erläuterung und Umsetzung von Wissensbeständen aus einem komplex strukturierten Bereich ist, der nach gesellschaftlicher Übereinkunft als „fachlich“ gilt. Am Erreichen des jeweiligen praktischen Ziels andererseits orientieren sich die Regeln, aus denen sich der Grad an Explizitheit und damit auch die Komplexität des sprachlichen Anteils der Interaktion ergeben. Die Beschäftigung mit Fachkommunikation hat den Fokus zumeist stark auf die Bereiche gerichtet, in denen die repräsentierte Komplexität ihren Widerhall in einer entsprechend komplexen sprachlichen Strukturierung findet. Man sollte aber auch über die andere Seite nachdenken. Fachlich sind Praktiken, wenn sie professionelles Handeln realisieren, das durch professionelles Wissen und Können gestützt ist.

Ein schönes und nicht ganz ernsthaftes Beispiel des Aufeinandertreffens der beiden ,extremen“ Vorstellungen von der sprachlichen Art fachlicher Interaktion, am theoretischen und am praktischen Ende, stellt uns die folgende Karikatur vor Augen. Sie stammt aus dem „Simplicissimus“, der bekanntesten deutschen satirischen Zeitschrift des frühen zwanzigsten Jahrhunderts.

(6) (Stimme von oben:) ,Laß abi - hiiah - laß abi, sag “ $i$-nach drah'n mer's anders ,rum!“--- „Es ist doch völlig unfaßlich, Herr Kollega, daß eine so komplizierte und exakte Arbeit in dieser Sprache zustande kommen kann!"

In der Sache geht es, wenn man es im Sinne unseres Beitrags formulieren will, um die Umsetzung ingenieurwissenschaftlichen und bauphysikalischen Wissens in Praxis: ein großer Stahlbrückenteil wird eingehoben. Das ist zweifellos eine Arbeit für fachlich qualifizierte Arbeiter. Sie kennen das Verfahren und seine möglichen Schwierigkeiten, sie greifen nur sprachlich ein, wo in diesem Sinn das reine Tätigwerden für eine verlässliche Realisierung der Praktik nicht mehr ausreicht. Das führt zur Meisterung komplexer Handlungsabläufe, bei der nicht viele Worte gemacht werden, eher indexikalische Hinweise in sprachlicher Kurzform gegeben werden. Dass das noch dazu auf Bairisch geschieht, macht sozusagen ihre sprachliche Restriktion in den Augen einer beobachtenden Gruppe von Professoren nicht besser. Die Distanz zu den Erwartungen universitärer Wissenschaftlichkeit könnte größer kaum sein.

Das professorale Staunen mag einen amüsieren. Man sollte sich aber nicht zu sehr über den beschränkt akademischen Blick wundern, der fachliche Adäquatheit mit hoher Explizitheit, Komplexität und Verbalität verbindet, ist es doch diese Fehlsicht, die auch der frühen Soziolinguistik mit der Identifikation des sogenannten restringierten Codes unterlaufen ist. Jedenfalls zeigen sie sich als Beobachter dieser Szene überrascht, dass so etwas Kompliziertes mit solch reduzierten sprachlichen 


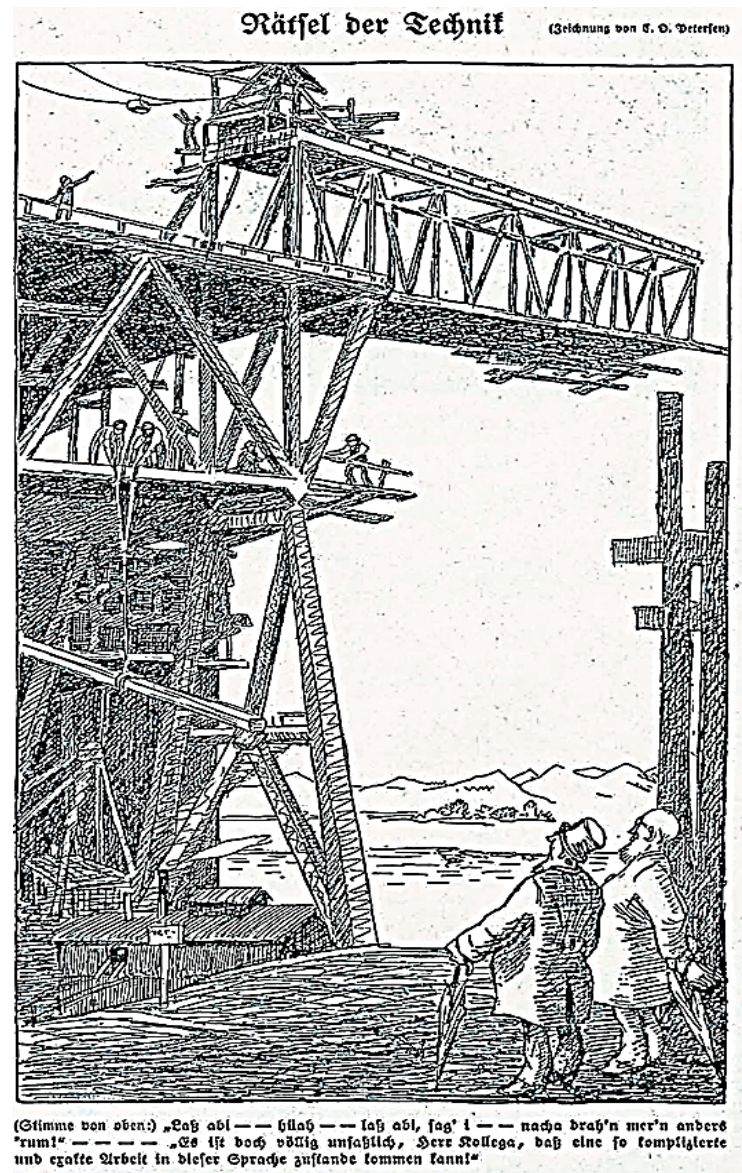

Simplicissimus 16. 9. 1912, S. 393 (URL 1)

Mitteln bewirkt werden könne. Sie assoziieren fachliche Praktik mit komplexer Sprachlichkeit. Erkennbar werden die Verhältnisse zwischen der Komplexität der Aufgabe, der sprachlichen Ausgestaltung und dem Erfolg der Interaktion an den Kriterien eines anderen Subsystems gemessen. Der Erfolg der Arbeit liegt hier in einer praxisbezogenen Reduktion fachlicher Komplexität, die normalerweise in einem intermedialen System geleistet wird, die auf erfolgreiche Anwendung zielt. Hier bleibt die fachliche Komplexität versteckt, Fachlichkeitskriterium dürfte sein, dass sie bei Bedarf (etwa Störungen der Abläufe) explizit gemacht werden könnte (s. dazu z. B. Deppermann 2018:71).

Meisterlehre von der einen zur anderen Stufe der fachlichen Bewältigung der Welt bzw. eines arbeitsfähigen Modells sichert hier im Wesentlichen die fachliche Kontinuität über die Subsystemfolge hin (zum Konzept s. Sennett 2008:286-291). Nun ist das bei so direkt in Welt umsetzbaren fachlichen Erkenntnissen und ihren Ergebnissen relativ einfach, schwerer ist das in theoretischen Wissenschaften oder insbesondere auch in den Geisteswissenschaften.

\section{Fächer und Fachlichkeit}

\subsection{Ansprüche an fachliche Interaktion}

Wenn so eine Menge von einschlägigen Praktiken die fachliche Tätigkeit und das fachliche Sprechen ausmachen, fragt man sich, was ein Kriterium dafür ist, ein Fach zu sein. Diese Frage wird 
in der Regel durch einen gewissen Konsens darüber entschieden, was Dinge sind, die als fachlich gelten. Dabei stellt sich bei der Durchsetzung neuer Theorien, Methoden und auch Techniken immer einmal wieder die Frage nach der Qualität des Fachlichen. Häufig gilt die Institutionalisierung in einigermaßen offiziellen Kontexten als ein Kriterium. In den Wissenschaften kann das ein externes Signal wie die universitäre Verankerung mit ihren beruflichen Möglichkeiten sein. Eher interne Anzeichen einer Prätention auf Fachlichkeit wären es, wenn in den Wissenschaften etwas handbuchartig erfasst wird, wie etwa jetzt die verschiedenen Gebiete, die in der Handbuchreihe, Sprache und Wissen 'versammelt sind. Auf einer anderen Ebene ist es für die Teilnahme am fachlichen Diskurs kennzeichnend, dass man die Sozialsymbolik der jeweiligen Bereiche kennt. So gilt in den Wissenschaften die Existenz von Ausdrucksformaten, die als wissenschaftlich anerkannt sind, durchaus als Nachweis angemessener Fachlichkeit eines Bereichs, und ihre Beherrschung als ein Signal fachlicher Zugehörigkeit.

Eine andere Art von Schwierigkeit stellt sich, wenn man zu bestimmen versucht, wo man denn die Grenze der Fachlichkeit bzw. der Fachlichkeit eines Diskurses ansetzt. Im Prinzip gilt zumindest, dass man, um fachlich und auf fachliche Weise mitsprechen zu können, die entsprechenden Dinge professionell gelernt haben soll, und dass man sie im Idealfall auch als Profession betreibt. Kann ein „Amateur“ eine fachliche Interaktion bestreiten? Über längere Zeit, seit der Professionalisierung der meisten Tätigkeiten, auch: der „Wissenschaft als Beruf“, würde man annehmen, dass das nicht der Fall ist. Und für viele der sogenannten ,harten“ Wissenschaften scheint das auch weithin unstrittig zu sein. Und wenn so etwas denn doch einmal der Fall ist, erscheint es weithin als eine aus dem normalen Rahmen fallende Erscheinung. Um hier ein Beispiel aus dem Bereich der Sprachwissenschaft zu bringen. Es gibt einen nun an der Universität Regensburg angestellten und in diesem Bereich praktisch sehr kompetenten Berater in Fragen der Rechtschreibung, der sich aus intensiver Neigung heraus zu einem Spezialisten in diesem Gebiet entwickelt hat, was vielleicht auch zeigt, dass anwendungsnahe Subsysteme solchen Erscheinungen eher offenstehen.

(7) Christian Stang, im Jahr 1975 in Regensburg geboren und Postler von Beruf, liebt die deutsche Rechtschreibung. Diese Art Liebe ist wahrscheinlich ähnlich selten zu konstatieren wie die zur Arbeit des Deutschen Instituts für Normung oder zur Straßenverkehrszulassungsordnung. Bei Stang begann sie irgendwann in den ersten Jahren seiner Schulzeit. Er wünschte sich einen Duden zum Geburtstag. Um seinen 15. Geburtstag herum fing er an, regelmäßig die Sprachfachzeitschrift „Muttersprache“ zu lesen. Mit 18 wies er den Autoren eines Rechtschreibratgeberbuches mehrere Fehler nach. Der Verlag lud ihn daraufhin ein, selbst ein Rechtschreibbuch zu schreiben. (URL 2)

Dennoch ist der „fachliche“ Status solcher Aktivitäten umstritten und spielt eine erhebliche Rolle bei der Diskussion um Status, Anspruch und Realität der Erscheinungen und Initiativen, die man seit einiger Zeit unter dem Schlagwort citizen science diskutiert (vgl. z. B. Finke 2016). Vielleicht ist aber der in diesem Beispiel angesprochene Fall weniger schwierig, da er als eine Art angewandter Wissenschaft verstanden werden kann, die sich an den prinzipiellen Ansprüchen der professionell gestützten Fachlichkeit orientiert. Kritischer ist da die Auseinandersetzung der Sprachwissenschaft mit Sprachkritikern, die von außerhalb der Profession kommen - wie zum Beispiel dem vor einigen Jahren sehr erfolgreichen und populären Bastian Sick - die zeigt, dass auch das nicht so einfach ist (vgl. zu verschiedenen Positionen in dieser Frage Eichinger 2009).

\subsection{Fächer und ihre Wortschätze}

Es gibt eine erhebliche Breite dessen, was man als fachlich betrachten kann. Und wenn man die Existenz und Beherrschung und den kompetenten Umgang von fachlichen Terminologien als ein Kriterium betrachtet, ist es bemerkenswert, dass ausweislich der Untersuchung Wolfgang Kleins (2013:34) im ersten Akademiebericht zum Zustand der deutschen Sprache die Größenordnung des Wortschatzes der deutschen Sprache im letzten Jahrhundert enorm zugenommen hat. 
(8) Wortschatzgröße

$\begin{array}{llll}\text { Zeitspanne } & 1905-1914 & 1948-1957 & 1995-2004 \\ \text { Lemmata } & 3715000 & 5045000 & 5328000\end{array}$

Enthält ein Textcorpus der deutschen Sprache von einer Größe etwa einer Milliarde Textwörter zum Anfang des 20. Jahrhunderts etwa 3,7 Millionen verschiedene Wörter, so an dessen Ende etwa 5,3 Millionen, also zwischen einem Viertel und einem Drittel mehr. Dabei erscheint zudem auffällig (Klein 2013:39), dass parallel die Quote verschiedener Wörter, die im Verlaufe von 10 Millionen Wörtern verwendet werden, in wissenschaftlicher Prosa und Gebrauchsprosa um 20 bzw. gut 30 \% zugenommen hat, in Zeitungen um etwa 30 \%, auf bei weitem die höchste Zahl von 85000 Einheiten.

(9) Verteilung (verschiedene Wörter pro 10 Mio. Wörter)

\begin{tabular}{llll} 
& $1905-1914$ & $1948-1957$ & $1995-2004$ \\
\hline Belletristik & 52700 & 57400 & 57000 \\
Zeitungen & 66500 & 68500 & 84800 \\
Wissenschaftliche Prosa & 64800 & 70800 & 76200 \\
Gebrauchstexte & 54500 & 66800 & 75900 \\
\hline
\end{tabular}

Der Zuwachs des Wortschatzes in dieser Zeit hängt eindeutig mit der fachlichen Ausdifferenzierung der sich modernisierenden Gesellschaften zusammen. Signifikant ist dafür gerade auch der Aufwuchs in den Zeitungstexten, die über die größten Teile des Jahrhunderts hin als die zentrale Vermittlerinstanz für die fachlichen Neuerungen und ihre sprachliche Fassung gewirkt haben. Natürlich: bevor das Internet kam - zu den Verhältnissen dort wissen wir noch nichts Rechtes. Man kann aber auf jeden Fall feststellen, dass damit eine nie gekannte Diversifizierungsmöglichkeit bei den Informationsmöglichkeiten wie Mitsprachemöglichkeiten des Einzelnen entstanden ist. Es ist damit auch die Entstehung von neuen Arten von fachlich ausgerichteten Communities verbunden, und neue Arten der Wissensvermittlung.

\section{Fachliches Wissen und der moderne Alltag}

\subsection{Fachlichkeit und gesellschaftliche Diversifikation}

Diese Diversifizierung passt nun gut zu den gesamtgesellschaftlichen Entwicklungen der letzten Jahrzehnte, wie sie die Kultursoziologie seit den Arbeiten von Gerhard Schulze (1992) und Ulrich Beck (1986) beobachtet und in Konzepten wie dem der Erlebnisgesellschaft oder der Risikogesellschaft beschrieben hat. Was diese Konzepte im Groben gemein haben, ist, dass sie eine Ablösung des Individuums von traditionalen Bindungen und die „Neuorganisation“ in Lebensstilbereichen als zentrale gesamtgesellschaftliche Entwicklung beschreiben. Zu diesen Lebensstilen gehört auch die Orientierung an jeweils dazu passenden fachlichen Interessen. Das fällt nun damit zusammen, dass wir zumindest im bundesdeutschen Teil des deutschen Sprachraums seit den 1970er Jahren insgesamt einen Trend zur Verfachlichung der Interaktion beobachten. Das hat damit zu tun, dass die 1960er Jahre in Deutschland eine Phase dramatischer Modernisierung und Bildungsexpansion waren; so fällt etwa der Beginn der Welle der Neugründungen von Universitäten in diese Zeit; sie führte z.B. dazu, dass sich in Bayern in diesem und dem folgenden Jahrzehnt die Zahl der Universitäten von anfänglich vier auf 10 erhöhte, mit der notwendigen Folge einer ebenso dramatischen Erhöhung der Studentenzahl. Das Jahr 1968, das in diesem Kontext mit Studentenrevolten in Verbindung gebracht wird, stellt dabei nur eine Etappe dar, allerdings eine insofern bedeutsame, als mit ihr auch das alltägliche Leben in einen wissenschaftlich-gesellschaftlichen Diskurs eingebunden wurde, eine Entwicklung, die die eigentlichen Folgen dieser Bewegung bei weitem überlebte. Sie führte einerseits zu einer Professionalisierung vieler Bereiche, die bis dahin als Elemente des Alltags 
betrachtet worden waren, andererseits zu einer zumindest lexikalisch-terminologischen Annäherung der professionellen und der alltäglichen Darstellung, in gewissen Bereichen, etwa am Tabubereich der Sexualität war es erst dieser Verfachlichungsschub, der eine allgemeine diskursive Entfaltung des Themas erlaubte. Da es sich in diesem Bereich zweifellos um alltagsnahe, wenn auch tabubehaftete Praktiken handelt, führt das dazu, dass sich hier eine neue sprachliche Zwischenwelt von öffentlichkeitsrelevanter Fachlichkeit herausbildet, der seine Elemente aus einer strikten fachlichen Sprachwelt, und aus der diskursiven Welt sprachlich aktueller Narrative (,kulturelles Repertoire“, „gender", „hegemoniale Kultur" usw.) entnimmt. Und so kann ein soziologischer Fachtext zu diesem Thema heute zum Beispiel folgendermaßen aussehen:

(10) Vieles spricht dafür, dass gerade in der neuen Mittelklasse ein breiteres kulturelles Repertoire von Geschlechtermodellen des Weiblichen und des Männlichen zur Verfügung steht, aus dem man nun jeweils sein eigenes Geschlechtsprofil komponiert. [...] Die spätmoderne Gender-Kultur hält infolgedessen ein Portfolio von Gender-Accessoires bereit, beispielsweise das Muster der , empathischen Frau' wie das der, toughen Frau', den ,neuen Mann '(auch: die ,neuen Väter'), aber auch den maskulinen Mann. Es ergibt sich damit ein Repertoire für die einzelne Kombination passender Geschlechtlichkeit, das allerdings in der hegemonialen Kultur offenbar weiterhin Grenzen des gesellschaftlich Akzeptierten enthält (die , vulgäre Frau' oder der ,effeminisierte Mann'). (Reckwitz 2017:349-350).

Dieser Text ist im Kern fachlich, die als Zitate gekennzeichneten Elemente sollen dem weniger fachlich informierten Leser den fachlichen Kontext erleuchten. Vieles aus diesem sozialwissenschaftlichen Modell einer individuellen Konstruktion von Geschlechts-Identitäten prägt auch den allgemeinen Diskurs über Sexualität, und ist in dieser Form zum Bestandteil eines modernen urbanen liberalen Lebensentwurfes und im Wesentlichen ein Signal eines entsprechenden sozialen Milieus geworden.

\subsection{Citizen Science und der Experte}

Man kann es für eine Folge dieser Entwicklungen halten, dass sich - nicht zuletzt im Kontext der Vernetzung im Internet - Gruppen fachlich interessierter Personen miteinander verbinden und interagieren, in denen sich eine ganz spezifische Expertise versammelt, ohne dass sie notwendigerweise durch professionelle Fachlichkeitsansprüche gestützt wäre. Dem entspricht vielleicht die zunehmende Bedeutung des Experten als einer Person, die geeignet erscheint, in bestimmten Problemlagen Wissen präsentieren zu können, das bei der Entscheidungsfindung hilft. Obwohl jeder Wissenschaftler zweifellos ein Experte für bestimmte Dinge in seiner Wissenschaft sein sollte, ist nicht jeder Wissenschaftler ein guter Experte: so sind oder waren in der öffentlichen Wahrnehmung zum Beispiel fast häufiger Journalisten als Islamwissenschaftler Experten für Nahostfragen. Das hat auch damit zu tun, dass zur Fachlichkeit des Wissenschaftlers immer der Zweifel gehört, der Gedanke, dass es möglicherweise auch anders sein könnte, beim Experten ist dagegen eher die handlungsleitende Eindeutigkeit verlangt. Und so haben wir es im obigen Fall des zum Rechtschreibspezialisten gewordenen Postbeamten (s. Beleg (7)) zweifellos mit einem Experten zu tun, der auch als solcher fungiert, und der auch weit in die fachlichen Weisen eingedrungen ist, in denen dieses Thema behandelt wird, so dass seine Texte sicher einer „angewandten“ Ebene von Fachlichkeit entsprechen, wie sie auch die üblichen Codices zur Rechtschreibung pflegen. Nun ist die Expertise im Fall Rechtschreibung wegen der öffentlichen Meinung zu diesem Thema zwar ein Thema, in dem man sich als Fachmann und Experte profilieren kann (was offenbar nicht ganz dasselbe ist), es ist allerdings insgesamt weniger geeignet, den Dienst zu erfüllen, den Spezialisierungen in den prägenden Milieus von postmodernen Gesellschaften offenbar nun bevorzugt spielen, sich auf diese Weise als ein interessantes, idealerweise ,einzigartiges“ Individuum zu präsentieren, wie das etwa in den Diskursen über Geschlecht und Gender der Fall ist (s. dazu die Beiträge in Ewels/Plewnia 2020). Das ist es ja, was die von der Kultursoziologie in den letzten Jahren in den Vordergrund 
gerückte „Singularität“ meint. Mit dieser Form sozialer Präsentation, die im Wesentlichen von jüngeren akademisch gebildeten urbanen Personen getragen wird, ist prinzipiell eine Diversifikation der Wissensbestände verbunden.

\subsection{Vom fachlichen Wissen zur Singularität}

Einen beliebten Bereich dieser lebensweltlich relevanten Diversifikation stellt zum Beispiel auch eine eigentlich so grundlegende Praktik wie das „Essen (und Trinken)“ dar. Über lange Zeit waren hier die damit beschäftigen Wissenschaften und die Alltagsklassifikation weit voneinander entfernt, und damit auch die entsprechenden Sprechweisen. Das sieht man recht schön, wenn man die typischen Kontexte des Lexems „essen“, das zweifellos auf eine alltägliche Praktik bezogen ist, und die von „Ernährung“, einem Wort mit einem Distanzhintergrund, miteinander vergleicht. Das sieht folgendermaßen aus:

(11) Kontexte

\begin{tabular}{|c|c|}
\hline essen & Ernährung \\
\hline 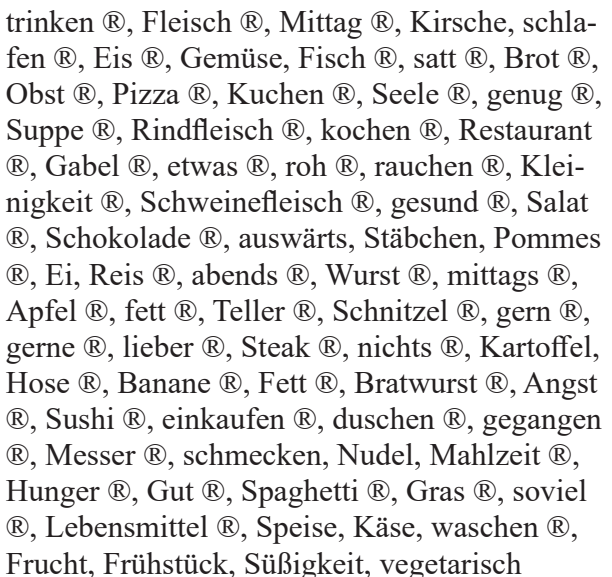 & $\begin{array}{l}\text { gesund, gesunde, Landwirtschaft, ausgewogen, } \\
\text { Gesundheit, künstlich, Verbraucherschutz, Be- } \\
\text { wegung, falsch, Bundesministerium, umstellen, } \\
\text { vegetarisch, Bundesforschungsanstalt, einseitig, } \\
\text { vollwertig, Bewegungsmangel, Bundesministerin, } \\
\text { richtig, Hauswirtschaft, fettarm, Gesellschaft, } \\
\text { vitaminreich, ungesund, achten, Rauchen, } \\
\text { ballaststoffreich, vernünftig, fettreich, Themen, } \\
\text { Lebensweise, Übergewicht, Entspannung, Kör- } \\
\text { perpflege, Fitness, Stress, bewusst, Ministerium, } \\
\text { Umstellung, umgestellt, Gemüse, Obst, Hygiene }\end{array}$ \\
\hline
\end{tabular}

Die Unterschiede sind augenfällig, die hochgestellten Zeichen beim Kontext von essen zeigen zudem, dass mit diesen Wörtern eine alltägliche Welt des Essens und Trinkens aufgerufen wird, während Ernährung zu einer auf fachliche Ausdrücke bezogenen Meta-Praxis der kritischen Reflexion dieser Handlung gehört. Der doch auch hohe Ausbau dieses Diskursnetzes zeigt seine gesellschaftliche Wirksamkeit, die sich bei einem genaueren Blick noch bestätigt. Man kann zum Beispiel die Einführung eines Berufsbilds des Ökotrophologen als Signal einer ersten Stufe gesellschaftsrelevanter Verwissenschaftlichung ansehen, und heutzutage ist die eigene Stellung an der Vorderfront der Ernährungsdiskussion, wie sie sich in diesem Kollokationenfeld zeigt, durchaus eine wichtige Karte im Spiel der Singularitäten, wie man unter anderem am Populärwerden von Termini und Konzepten bemerken kann, die hier sprunghaft in den öffentlichen Diskurs eindringen (was es dann logischerweise dem ,singulären“ Menschen nicht einfacher macht, singulär zu bleiben). Exemplarisch soll dafür die folgende Graphik aus google ngram stehen, die zeigt, wie früher allenfalls fachsprachliche Wörter ihren Wert in einem solcherart progressiven Diskurs gewinnen - und dann auch an Attraktivität wieder verlieren, hier im Kontext der Wörter für Lebensmittelunverträglichkeiten, ein Phänomen, das erkennbar lange Zeit in solch fester sprachlicher Form lediglich in fachlichen Praktiken eine Rolle spielte, um plötzlich eine relevante, in einen progressiv und positiv bewerteten Diskurs passende Rolle im Hinblick auf das Sprechen über das Essen und Trinken einzunehmen: 
(12) Singularitäten

\section{Google Books Ngram Viewer}
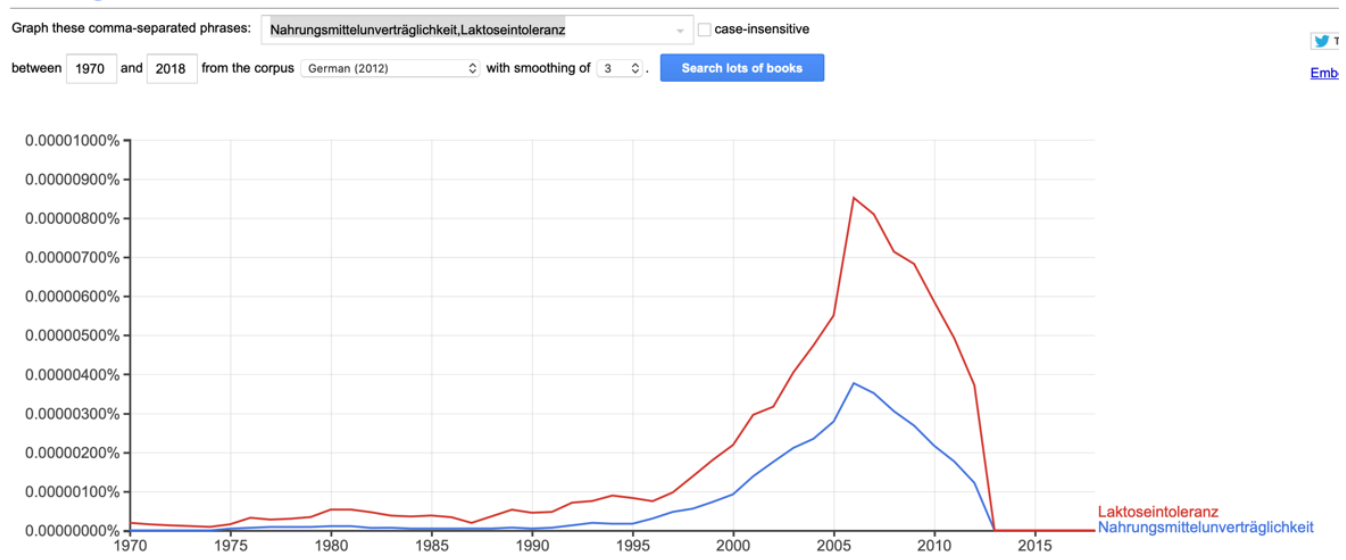

Ein weiteres Symptom für die Durchdringung der Fach- und der Laieninteraktion stellt die seit einigen Jahren aufgekommene Diskussion um „,citizen science“, die Bürgerwissenschaft, dar. Die Praxis in diesem Bereich produziert, wie man an entsprechenden sprachwissenschaftlichen Vorhaben sehen kann, etwa der Schweizerischen DilektÄpps (URL 3), derzeit noch nicht so sehr den eigentlichen Bürgerwissenschaftler (citizen science proper). Vielmehr profitiert sie davon - in diesem Fall durch die Nutzung von Apps -, dass aufgrund der qualitativen Veränderung im technischen Bereich die Möglichkeiten der Mitarbeit von Laien an von Wissenschaftlern konzipierten Projekten größer werden. Wenn sich allerdings die Idee in höherem Ausmaß umsetzen lässt, dass hier fachlich interessierte Laien eigene, andersartige Fragen einbringen sollten, die nicht Fragen der „klassischen“ Wissenschaft wären, die aber doch als wissenschaftlich gelten sollen, brächte das eine bemerkenswerte Verschiebung mit sich, was wir als fachliche Interaktion betrachten, und eben: wie das im Verhältnis zur fachsprachlichen Kompetenz steht.

\section{Vom Verschwinden der Fachlichkeit bei der Annäherung}

Dass der Wortschatz des Deutschen im letzten Jahrhundert dramatisch angewachsen ist, hat in der ersten Hälfte des Jahrhunderts sicher weithin damit zu tun, dass sich weitere fachliche Disziplinen entwickelt haben, die zur Kenntnis genommen werden. In den letzten Jahrzehnten kommt, wie angedeutet, eine generelle Verfachlichung des Sprechens über „Ausschnitte“ aus der Lebenswelt auf, verbreitet sich, und führt zu einer Diversifizierung der Lebenswelten, über die gesprochen werden soll. Nicht zuletzt das Internet ermöglicht die Entwicklung entsprechender Diskurswelten mit spezifischer Expertise. Verstärkt wird solch eine Tendenz durch den Trend zur Singularisierung in den meinungsführenden ,progressiven“ Milieus unserer Gesellschaft. Hier geht es um Sozialsymbolik, die unter anderem inhaltliche und formale Elemente von Fachlichkeit nutzt, ihren Hauptzweck aber nicht in der Fortentwicklung des Faches hat, was in anderer, aber ähnlicher Weise für ein öffentlichkeitwirksames Expertentum gilt.

Wenn man sich diesen Raum im Übergang vom Alltäglichen zum Fachlichen ansieht, scheint es mir vielversprechend, durch die Untersuchung der gesamten Interaktionsmodi, also durch das Ausgehen davon, was als eine fachliche Praxis gelten kann, eine klarere Sicht über die verschiedenen Kategorien von Handeln und Sprechen oder Schreiben in diesem Übergang zu gewinnen. 


\section{Literaturverzeichnis}

\section{Primärliteratur:}

EICHINGER, Anna (2018): Charakterisierung eines Mausmodells zur BH4-sensitiven Phenylketonurie. Dissertation. München: LMU, S. 1

ForsCHNER, Maximilian (1990): Über natürliche Neigungen. In: BuBNER, Rüdiger u.a. (Hrsg.): Die Trennung von Natur und Geist. München, S. 95.

Simplicissimus 16. 9. 1912, S. 393. Zugänglich unter URL 1: http://www.simplicissimus.info/uploads/tx_lombkswjournaldb/pdf/1/17/17_25.pdf; [18.03.2020].

RecKwITZ, Andreas (2017): Die Gesellschaft der Singularitäten. Berlin.

\section{Sekundärliteratur:}

AvH / Alexander von Humboldt Stiftung (Hrsg.) (2009): Publikationsverhalten in unterschiedlichen wissenschaftlichen Disziplinen. Beiträge zur Beurteilung von Forschungsleistungen. 2., erw. Aufl. (= Diskussionspapiere der Alexander-von-Humboldt-Stiftung 12). Bonn.

BaumanN, Klaus-Dieter (2018): Die fachkommunikativen Grundlagen der Verwaltungssprache: eine Annäherung an die interdisziplinäre Betrachtung von Fachlichkeit. In: VAŇKOvá, Lenka (Hrsg): Fachlichkeit und Fachsprachlichkeit in varianten Kontexten. Berlin, S. 35-47.

BECK, Ulrich (1986): Risikogesellschaft. Auf dem Weg in eine andere Moderne. Frankfurt a. M.

Brandom, Robert B. (2002): Expressive Vernunft. Begründung, Repräsentation und diskursive Festlegung. Frankfurt a. M.

Brandom, Robert B. (2001): Begründen und Begreifen. Eine Einführung in den Inferentialismus. Frankfurt a. M.

BüHLER, Karl (1999 [1934]): Sprachtheorie. Die Darstellungsfunktion der Sprache. Stuttgart. [Originalausgabe Jena 1934].

DepPermann, Arnulf (2018): Sprache in der multimodalen Interaktion. In: DepPermann, Arnulf / ReINEKE, Silke (Hrsg.): Sprache im kommunikativen, interaktiven und kulturellen Kontext. (= Germanistische Sprachwissenschaft um 2020 Band 3). Berlin; Boston, S. 51-85.

EHLICH, Konrad (2006): Mehrsprachigkeit in der Wissenschaftskommunikation - Illusion oder Notwendigkeit. In: EHLICH, Konrad / Heller, Dorothee (Hrsg.): Die Wissenschaft und ihre Sprachen (=linguistic insights 52). Bern u. a., S. 17-38.

EICHINGER, Ludwig M. (2005): Standardnorm, Sprachkultur und die Veränderung der normativen Erwartungen. In: EICHINGER, Ludwig M. / KALLMEYER, Werner (Hrsg.): Standardvariation. Wie viel Variation verträgt die deutsche Sprache? (= Institut für Deutsche Sprache Jahrbuch 2004), Berlin; New York, S. 363-381.

EICHINGER, Ludwig M. (2009): Vom rechten Deutsch. Wer darf die Sprache kritisieren? In: LIEBERT, Wolf-Andreas / Schwinn, Horst (Hrsg.): Mit Bezug auf Sprache. Festschrift für Rainer Wimmer (= Studien zur Deutschen Sprache 49). Tübingen, S. 201-217.

EICHINGER, Ludwig M. (2010): Textsinn und sprachliche Struktur. Oder: Ist es dem Vogel Textsinn egal, welchen strukturellen Käfig er sich gesucht hat? In: Foschi Albert, Marina / HePP, Marianne / Neuland, Eva / Dalmas, Martine (Hrsg.): Text und Stil im Kulturvergleich. München S. 154-169.

EICHINGER, Ludwig M. (2016): Praktiken: etwas Gewissheit im Geflecht der alläglichen Welt, in: Deppermann, Arnulf / FeILKe, Helmuth / Linke, Angelika (Hrsg.): Sprachliche und kommunikative Praktiken (= Institut für Deutsche Sprache Jahrbuch 2015) Berlin; Boston, S. VI-XIII.

EICHINGER, Ludwig M. (2017): Deutsch als Wissenschaftssprache. In: Moraldo, Sandro (Hrsg.): Die deutsche Sprache in Italien - Zwischen Europäisierung und Globalisierung (Duisburger Arbeiten zur Sprach- und Kulturwissenschaft 120). Frankfurt a. M. u. a., S. 45-70.

EICHINGER, Ludwig M. (2020; i.Dr.): Ökonomie der Mehrsprachigkeit. In: Tender, Tõnu / EICHINGER, Ludwig M. (Hrsg): Language and Economy. Proceedings of the 17th Annual conference of EFNIL in Tallinn on 9, 10 and 11 October 2019. Budapest. 
Ewels, Andrea-Eva / Plewnia, Albrecht (Hrsg.) (2020): Themenheft „Sprache und Geschlecht“ Beiträge zur Gender-Debatte (= Muttersprache 130, 2020, H.1). Wiesbaden.

FANDRYCH, Christian (2018): Wissenschaftskommunikation. In: DePPERMAnN, Arnulf / ReIneKe, Silke (Hrsg.): Sprache im kommunikativen, interaktiven und kulturellen Kontext. (= Germanistische Sprachwissenschaft um 2020 Band 3). Berlin; Boston, S. 143-167.

Finke, Peter (2016): Citizen Science und die Rolle der Geisteswissenschaften für die Zukunft der Wissenschaftsdebatte. In: Oswald, Kristin / Smolarski, René (Hrsg.), Bürger - Künste - Wissenschaft: Citizen Science in Kultur- und Geisteswissenschaften. Gutenberg, S. 31-56.

GIESECKE, Michael (1998): Der Buchdruck in der frühen Neuzeit. Eine historische Fallstudie über die Durchsetzung neuer Informations- und Kommunikationstechnologien. Frankfurt a. M.

HoFFMANN, Lothar / KALVERKÄMPER, Hartwig (1998): Forschungsdesiderate und aktuelle Entwicklungstendenzen in der Fachsprachenforschung. In: HofFMAnN, Lothar et al.: Fachsprachen (=HSK 14. 1.). Berlin; New York, S. 355-372.

KLEIN, Wolfgang (2013): Von Reichtum und Armut des deutschen Wortschatzes. In: Deutsche Akademie für Sprache und Dichtung/ Union der Akademien der Wissenschaften (Hrsg.): Reichtum und Armut der deutschen Sprache. Erster Bericht zur Lage der deutschen Sprache, Berlin; Boston, S. 1-55.

Linke, Angelika (2015): Sprachreflexion und Menschenbild. Entwürfe zum Verhältnis von Sprachlichkeit und Sozialität. In: EIchInger, Ludwig M. (Hrsg.): Sprachwissenschaft im Fokus. Positionsbestimmungen und Perspektiven (= Institut für Deutsche Sprache Jahrbuch 2014) Berlin; Boston, S. 9-42.

Schulze, Gerhard (1992): Erlebnisgesellschaft: Kultursoziologie der Gegenwart. Frankfurt; New York.

SEnNET, Richard (2008): The Craftsman. New Haven; London.

TAYLOR, Charles (2016): The Language Animal. The Full Scope of the Human Linguistic Capacity. Cambridge/Mass; London.

WeINRICH, Harald (1973): System, Diskurs, Didaktik und die Diktatur des Sitzfleisches. In: MacIEJEWsKI, Franz (Hrsg.): Theorie der Gesellschaft oder Sozialtechnologie (=Theorie-Diskussion Supplement 1). Frankfurt am Main, S. 145-161.

WitTGEnStern, Ludwig (1971): Über Gewißheit. Frankfurt am Main.

ÁGEL, Vilmos (2015): Die Umparametrisierung der Grammatik durch Literalisierung. Online- und Offlinesyntax in Gegenwart und Geschichte. In: EIcHINGER, Ludwig M. (Hrsg.): Sprachwissenschaft im Fokus. Positionsbestimmungen und Perspektiven (= Institut für Deutsche Sprache Jahrbuch 2014) Berlin; Boston, S. 121-156.

\section{Internetquellen:}

URL 1: http://www.simplicissimus.info/uploads/tx_lombkswjournaldb/pdf/1/17/17_25.pdf

URL 2: https://www.tagesspiegel.de/politik/ein-mann-aus-woertern/7571328. $\bar{h}$ tml [18.03.2020].

URL 3: https://www.schweiz-forscht.ch/de/citizen-science-projekte/item/251-dialaekt-aepps [18.03.2020]. 